\title{
Validity of Hand-to-Foot Measurement of Bioimpedance: Standing Compared with Lying Position
}

\author{
Elaine C. Rush,* Jennifer Crowley, * Ismael F. Freitas, $\dagger$ and Amy Luke $\neq$
}

\begin{abstract}
RUSH, ELAINE C., JENNIFER CROWLEY, ISMAEL F. FREITAS, AND AMY LUKE. Validity of hand-to-foot measurement of bioimpedance: standing compared with lying position. Obesity. 2006;14:252-257.

Objective: To assess the reliability of the standing measurement of hand-to-foot bioimpedance compared with measurements made in the lying position.

Research Methods and Procedures: In 205 volunteers 6 to 89 years of age, 111 males and 94 females from six ethnic groups, effects of posture, time, and age on hand-to-foot resistance were studied over a range of body size. The effect of time in a position on resistance was also recorded in a small subset $(n=10)$, and repeat measurements over 3 days at the same time of the day were recorded in another subset $(n=12)$.
\end{abstract}

Results: Lying impedance was consistently higher than standing, with the relationship (resistance lying/resistance standing) for the children (5 to 14 years) being 1.031, progressing to a ratio of 1.016 in those $>60$ years. The time spent static in either position did change resistance measurements-a decrease of up to $9 \Omega$ (mean $5 \Omega, 1.0 \%$ ) over 10 minutes of standing and an increase of up to $7 \Omega$ (mean $3 \Omega, 0.7 \%$ ) with lying.

Discussion: In the field, measurements of hand-to-foot bio-

Received for review June 20, 2005

Accepted in final form December 2, 2005.

The costs of publication of this article were defrayed, in part, by the payment of page charges. This article must, therefore, be hereby marked "advertisement" in accordance with 18 U.S.C. Section 1734 solely to indicate this fact.

*Division of Sport and Recreation, Faculty of Health and Environmental Sciences, Auckland University of Technology, Auckland, New Zealand; †UNESP São Paulo State University, Department of Physical Education, Campus Presidente Prudente, Sao Paulo, Brazil; and ¥Department of Preventive Medicine \& Epidemiology, Loyola University Medical School, Maywood, Illinois.

Address correspondence to Elaine Rush, Division of Sport and Recreation, Faculty of Health and Environmental Sciences, Auckland University of Technology, Akoranga Campus, 90 Akoranga Drive, Northcote, Auckland, New Zealand.

E-mail: elaine.rush@aut.ac.nz

Copyright (C) 2006 NAASO impedance can be made in the standing position, and, with appropriate adjustment, previously validated recumbent equations can be used. Given that errors in the measurement of height and weight also affect the reliability of the derivation of body fat from bioelectrical conductance, the errors that may arise from a more practical standing measurement rather than lying are minimal.

Key words: single frequency bioimpedance analysis, position, lying, standing, correction factor

\section{Introduction}

Body impedance is directly related to water distribution and hydration of the body. In normally hydrated people, this relationship can be extended to fat-free mass and fat mass. The common and recommended (1) method of measurement of body impedance and that for which many population-specific prediction equations have been validated is for the subject to be in a recumbent position with arms and legs slightly abducted and electrodes on the hand and foot. In 2004, the principles and methods for bioelectrical impedance analysis (BIA) ${ }^{1}$ were reviewed (2), and guidelines for clinical practice (3) were updated, with hand-to-foot assessments in the lying position being the most often published. However, there is no common validation equation for estimation of body composition from bioimpedance measurement; therefore, it is important that the BIA equation chosen has been verified against reference methods in the population being studied. In the field, it is more difficult to provide the ideal conditions, because subjects can object, feel insecure, or be embarrassed if asked to lie on the floor, ground, or a raised platform, and it may be difficult to provide a wide enough bed or an appropriate place to put the head. It also is not easy physically for some people to lie down and sustain a static position or to rise because of joint, respira-

${ }^{1}$ Nonstandard abbreviation: BIA, bioelectrical impedance analysis. 
tory, or body size problems. The person doing the measurement also needs to be able to help people up and down and kneel on the ground if that place is used.

The ambient temperature of the room and the skin can also be a problem, especially because prolonged lying or standing may cause cooling. Gudivaka et al. (4) found that impedance varies inversely with skin temperature, i.e., impedance increases with a lowering in temperature and decreases with a rise in skin temperature, and that this change in conductance seems to be caused by a change in the conductance of the skin and not a change in cutaneous blood flow or compartmental fluid shifts. Therefore, the warmth, time, and comfort in a position to achieve a reliable and stable reading also needs to be considered.

An initial report (5) noted that standing resistance could be multiplied by $2.3 \%$ to make it comparable with published recumbent measurements. In a pilot study (6) in elderly people, we developed this observation further and suggested that the measurement of bioimpedance in the standing position, with an appropriate adjustment, can be a good alternative to lying down. So that previously validated equations for hand-to-foot bioimpedance measurement of body water may be modified for use in the standing position, the aims of this study were to 1) investigate the effects of posture, time, sex, and age on the relationship between standing and lying resistance and 2) provide evidence for a more practical and reliable method of measuring hand-to-foot bioimpedance.

\section{Research Methods and Procedures}

Two hundred five (94 female and 111 male) ambulant subjects 6 to 89 years of age participated. Twenty-five subjects were drawn from an ongoing study with elderly Asian Indians. A further 180 subjects were recruited as a convenience sample at both the Auckland University of Technology and local community groups. Exclusion criteria included conditions that did not allow the subject to stand or remain still, skin conditions that did not allow the placement of the electrodes, scarring that affects the skin impedance, amputation of the right arm or leg, and ascites or edema. The Auckland University of Technology Ethics Committee approved the study. All adult participants or children's caregivers signed informed consent.

Background information on participant's age, sex, ethnicity, and time since food was last eaten and the bladder last voided was recorded before testing. Standing height was measured, without shoes, in duplicate to the nearest $0.1 \mathrm{~cm}$ with a stadiometer. Body weight was measured without shoes and in light clothing in duplicate to the nearest $0.1 \mathrm{~kg}$. Waist circumference was measured in duplicate to the nearest $0.1 \mathrm{~cm}$, using a non-stretch inelastic tape placed at the midpoint between the lower rib and upper iliac crest. The temperature of the room was recorded, and subjects stood barefooted on an insulating mat. Subjects moved freely for the same period of time between measurements. All measurements were performed by trained researchers.

BIA measurements were made using a single-frequency (50 kHz) battery-operated bioimpedance analyzer (BIM4; Impedimed, Queensland, Australia). Areas of the right hand and foot where the electrodes, Red Dot 2330 or Impedimed Body Comp BIA electrodes, were to be placed were cleaned with alcohol. The current electrodes were placed on the foot, over the distal portion of the second metatarsal, and on the hand on the distal portion of the second metacarpal. The sensing electrodes were placed at the anterior ankle between the tibial and fibular malleoli and at the posterior wrist between the styloid processes of the radius and ulna. The bioimpedance measurements were recorded first with people standing (S1) without moving, with arms and legs slightly abducted so that they were not touching. Then the participants were asked to lie down in a supine position (L) on the floor or a bed in a similar position and then again to assume a standing position (S2). In each position, the measurements were repeated until they were stable to within 1 $\Omega$ (usually up to three times within an interval of $20 \mathrm{sec}-$ onds), and the average value was used in calculations.

In a subset of volunteers, 22 measurements were made over 23 minutes to assess the effect of time in a position on the measurements. Before and between the standing and lying positions, the subjects walked around to ensure that fluid distribution was comparable before each position was assumed.

At the same time of day for 3 consecutive days, 12 subjects (6 female and 6 male; 7 to 54 years of age) had the measurements repeated by the same researcher. The coefficients of variation of weight, height, and waist were $0.63 \%, 0.02 \%$, and $1.30 \%$, respectively. The daily variations of resistance measures of standing, lying, and standing again were $1.07 \%, 1.56 \%$, and $3.20 \%$, respectively.

Data are presented as means and SD. The Bland Altman method (7) was used to test the bias of measurement of resistance of standing vs. lying. The relationship of lying to standing with age was assessed using the Pearson correlation coefficient and also by grouping by age. The ratio of lying to standing was calculated by age group to provide a correction factor to adjust standing resistance measurements to equate with lying. Comparison of readings corrected for the standing position with lying was made using a paired Student's $t$ test.

\section{Results}

Subjects were drawn from six ethnic groups: European (153), Maori (8), Pacific (8), Indian (26), Asian (8), and other (4), representative of the diverse Auckland population. Subject characteristics are shown in Tables 1 and 2 and represented a wide range of weight (21.0 to $121.2 \mathrm{~kg}$ ), height $(116.5$ to $189.7 \mathrm{~cm})$, and waist $(32.7$ to $113.0 \mathrm{~cm})$ measurements and also fitness and fatness. 
Table 1. General characteristics and values of hand-to-foot resistance in standing and lying positions for males, grouped by age

\begin{tabular}{lcccc}
\hline & \multicolumn{4}{c}{ Age in years $(\boldsymbol{N})$} \\
\cline { 2 - 4 } & $\mathbf{5}$ to $\mathbf{1 4}(\mathbf{1 3})$ & $\mathbf{1 5}$ to $\mathbf{3 0}(\mathbf{4 3})$ & $\mathbf{3 1}$ to $\mathbf{5 9}(\mathbf{3 2})$ & $\mathbf{2 6 0}(\mathbf{2 3})$ \\
\hline Weight $(\mathrm{kg})$ & $49.9 \pm 25.1$ & $73.9 \pm 8.9$ & $83.0 \pm 12.8$ & $71.9 \pm 10.1$ \\
Height $(\mathrm{cm})$ & $152.8 \pm 17.0$ & $177.2 \pm 7.0$ & $174.0 \pm 7.0$ & $167.5 \pm 8.1$ \\
BMI $\left(\mathrm{kg} / \mathrm{m}^{2}\right)$ & $20.2 \pm 5.2$ & $23.5 \pm 2.2$ & $27.3 \pm 2.9$ & $25.6 \pm 3.2$ \\
Waist $(\mathrm{cm})$ & $66.7 \pm 20.2$ & $79.1 \pm 6.3$ & $90.4 \pm 9.3$ & $91.9 \pm 8.3$ \\
Stand $(\Omega)$ & $553.3 \pm 89.0$ & $454.4 \pm 42.7$ & $430.2 \pm 47.4$ & $514.6 \pm 82.4$ \\
Stand $2(\Omega)$ & $554.2 \pm 88.4$ & $455.4 \pm 42.8$ & $431.1 \pm 47.5$ & $509.9 \pm 79.7$ \\
Lying $(\Omega)$ & $573.3 \pm 93.0$ & $466.0 \pm 44.5$ & $437.6 \pm 48.7$ & $522.8 \pm 83.0$ \\
Diff L-S $(\Omega)$ & $20.0 \pm 6.2$ & $11.6 \pm 6.1$ & $7.4 \pm 4.7$ & $8.2 \pm 7.1$
\end{tabular}

Values are means $\pm \mathrm{SD}$.

Stand 1, first measure of the resistance in the standing position; Stand 2, second measure of the resistance in the standing position; Lying, value of the resistance in the lying position; Diff L-S, difference between the average resistance standing less resistance lying.

Time from the subject assuming the standing or lying position to readings that did not differ by $>1 \Omega$ averaged 75 seconds. Lying bioimpedance, on average, was $12 \Omega$ higher than standing bioimpedance (Figure 1). The second standing measurement was, on average, $1 \Omega$ lower than the first standing measurement. The difference between lying and standing increased as body resistance increased, but when examined as a percentage change in resistance, the increase was not linearly related to the body resistance and averaged $2.0 \pm 1.2 \%$. When the ratio of lying to standing was examined with respect to age (Figure 2), a significant de- crease of the ratio with increasing age was seen $\left(r^{2}=0.138\right.$ $p<0.00001)$. A negative relationship of the ratio to weight $\left(r^{2}=0.047 p=0.002\right)$ but not height $\left(r^{2}=0.011 p=\right.$ 0.134 ) was also observed. Grouping by age (children 5 to 14 years, young adults 15 to 30 years, middle-aged 31 to 59 years, and elderly $60+$ years) enabled the construction of a table of recommended factors to adjust the standing bioimpedance by to equate with that measured lying (Table 3 ). There was no significant difference between factors for males and females. When the standing resistance multiplied by the appropriate factor was compared with lying, the

Table 2. General characteristics and values of hand-to-foot resistance in standing and lying positions for females, grouped by age

\begin{tabular}{lcccr}
\hline & \multicolumn{4}{c}{ Age in years $(\boldsymbol{N})$} \\
\cline { 2 - 5 } & $\mathbf{5}$ to $\mathbf{1 4}(\mathbf{1 4})$ & $\mathbf{1 5}$ to $\mathbf{3 0}(\mathbf{1 8})$ & $\mathbf{3 1}$ to $\mathbf{5 9}(\mathbf{4 1})$ & $\mathbf{2 6 0}(\mathbf{2 0})$ \\
\hline Weight $(\mathrm{kg})$ & $30.9 \pm 7.4$ & $61.0 \pm 6.3$ & $66.9 \pm 15.2$ & $66.5 \pm 9.5$ \\
Height $(\mathrm{cm})$ & $136.0 \pm 13.0$ & $164.4 \pm 8.1$ & $162.1 \pm 6.1$ & $155.9 \pm 6.1$ \\
BMI $\left(\mathrm{kg} / \mathrm{m}^{2}\right)$ & $16.5 \pm 2.2$ & $22.6 \pm 1.9$ & $25.5 \pm 5.8$ & $27.4 \pm 3.6$ \\
Waist $(\mathrm{cm})$ & $56.0 \pm 5.2$ & $69.4 \pm 4.9$ & $77.8 \pm 10.8$ & $86.6 \pm 9.0$ \\
Stand $1(\Omega)$ & $669.9 \pm 65.0$ & $569.5 \pm 55.0$ & $564.5 \pm 66.2$ & $581.0 \pm 62.8$ \\
Stand $2(\Omega)$ & $671.0 \pm 64.7$ & $569.8 \pm 56.2$ & $566.6 \pm 66.3$ & $577.2 \pm 61.7$ \\
Lying $(\Omega)$ & $687.4 \pm 63.3$ & $581.3 \pm 57.5$ & $574.3 \pm 66.4$ & $590.2 \pm 62.8$ \\
Diff L-S $(\Omega)$ & $17.4 \pm 7.4$ & $11.8 \pm 5.1$ & $9.8 \pm 5.9$ & $9.2 \pm 5.8$
\end{tabular}

Values are means $\pm \mathrm{SD}$.

Stand 1, first measure of the resistance in the standing position; Stand 2, second measure of the resistance in the standing position; Lying, value of the resistance in the lying position; Diff L-S, difference between the average resistance standing less resistance lying. 


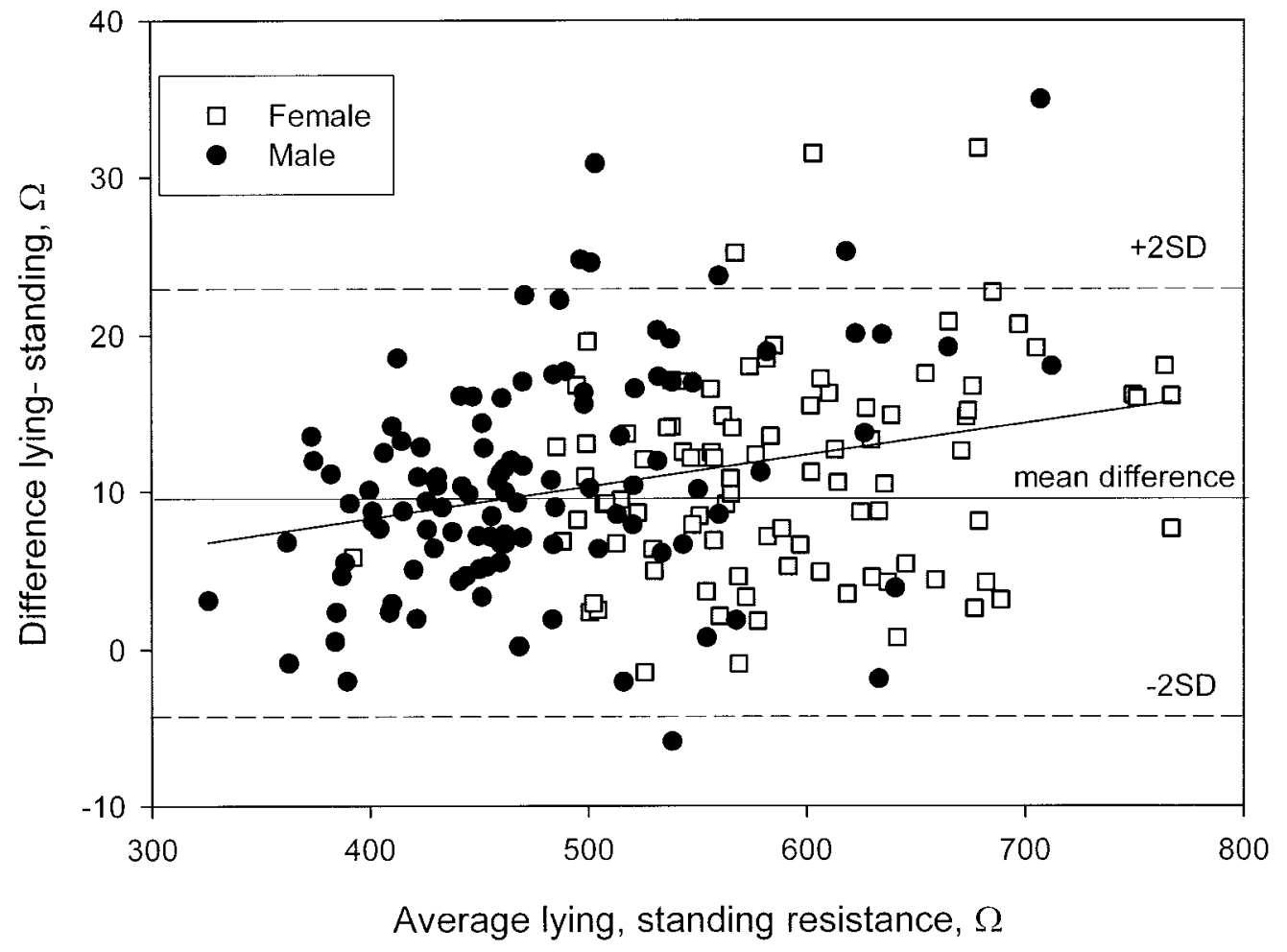

Figure 1: Bias related to position: lying and then standing hand-to-foot bioimpedance.

average difference was $0.2 \Omega$, with the $95 \%$ confidence limits of the difference being -1.02 to $0.63 \Omega$.

In 10 subjects, resistance was measured every half minute for 11 minutes standing and then 11 minutes lying. Standing resistance decreased with time and lying resistance increased in 10 of the 10 subjects measured in this way (Figure 3). The intra-individual change in one position within this time did not exceed $9 \Omega$ and was frequently less than this.

\section{Discussion}

When lying and standing bioimpedance hand-to-foot measurements at $50 \mathrm{kHz}$ were compared, we showed that there was a predictable change in body resistance for a wide range of bioimpedance, body size, age, sex, and diverse ethnicity. Small changes in resistance continued with prolonged lying or standing over 10 minutes and did not seem to stabilize at any point. In a small study (8), changes in impedance were tracked, and a 5\% rise after 10 minutes of lying was recorded, with a further $4 \%$ increase over 4 hours of sustained lying with no apparent period of stability. Using hand-to-foot and segmental multifrequency bioimpedance, it has been shown that changes in body position result in changes of resistance (9), with an average rapid increase of extracellular hand-to-foot resistance of $12.4 \%$ when going from sitting to lying and an increase in both arm and leg resistance with prolonged lying. Earlier work by Zhu et al. (10) and De Lorenzo et al. (11) in men also showed that extracellular and total resistance increased when going from standing to lying, whereas no significant changes were shown in women by others (12). We were unable to show any difference in the magnitude of the change between sexes. The best explanation of changes in resistance is hydrostatic fluid shifts. Quiet standing leads to a rapid reduction of plasma volume in the legs and lower arms, because in the early stages of standing, raised hydrostatic pressures in the limbs shifts fluid from the blood compartment into the interstitium, and venous return is reduced. In men (average weight, $78 \mathrm{~kg}$ ), from changes in hematocrit, it was determined that in the first 3 minutes of standing, $\sim 330 \mathrm{~mL}$ left the blood compartment, with the total loss of fluid from the blood stabilizing at $\sim 700 \mathrm{~mL}$ within the first 10 minutes of standing (13). Furthermore, in both men and women, single frequency bioimpedance over 12 hours in the lying position has been shown to increase, with the most rapid change taking place over the first 30 minutes (14). We showed in larger numbers that the magnitude of the resistance change with time is not sex-specific but does decrease with age, perhaps indicating age-related differences in vascular tone and permeability. 


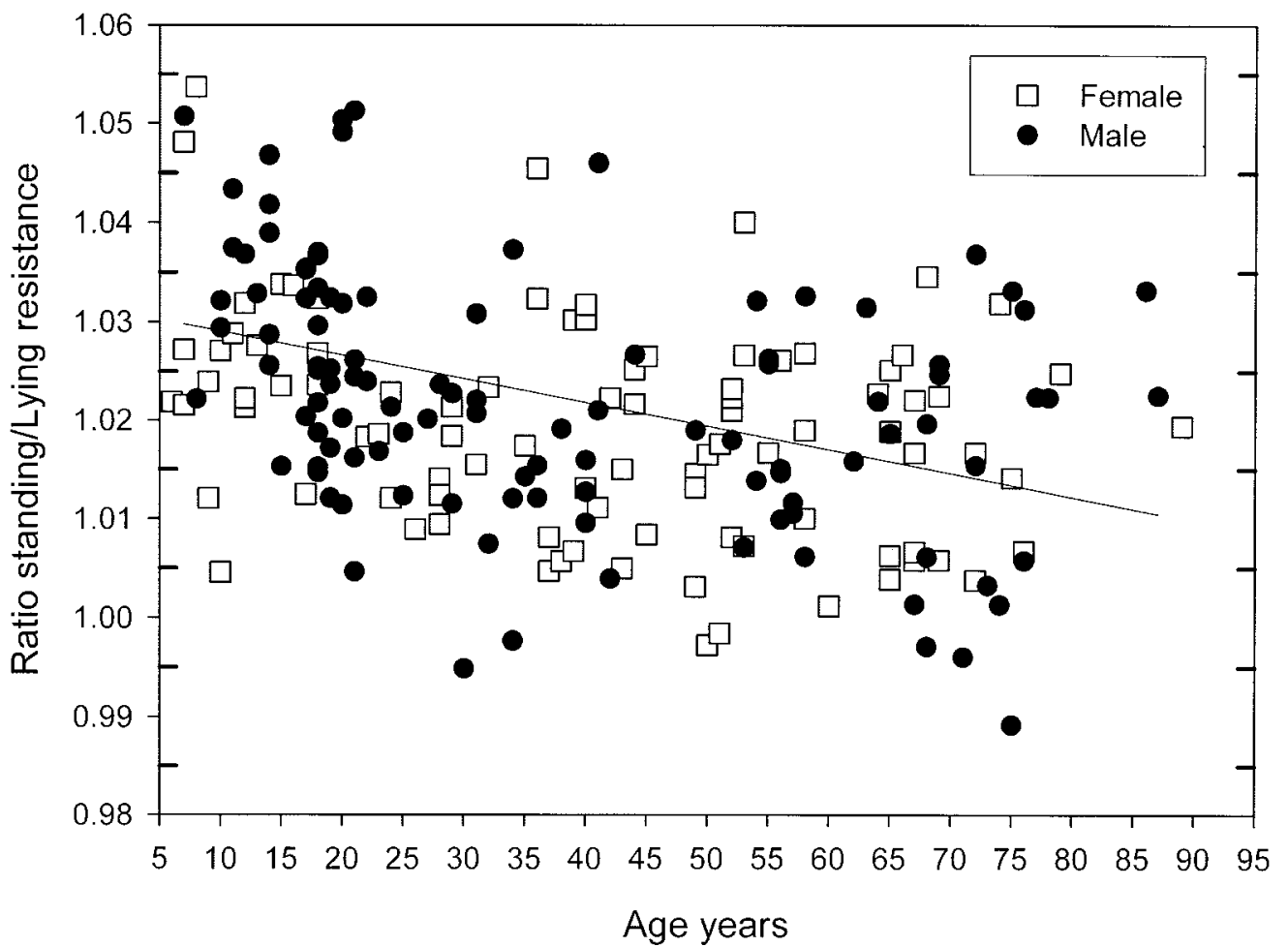

Figure 2: Relationship of standing to lying resistance ratio to age.

Day-to-day variation in resistance measurement is reported as ranging from $2 \%$ to $3.5 \%$ (2). Our coefficients of variation of measurement ( $1.1 \%$ to $3.2 \%$ ) are in this order of magnitude. In addition, the calculation of body fat from bioimpedance often uses both the height squared and the weight in the prediction equation-these add another $1 \%$ of error that may propagate the errors further. Additionally, making sure that the bladder is empty, that food or fluid have not recently been drunk, that the limbs are adequately abducted, that the subject is relaxed, and that the hands and feet are warm will reduce variation and improve accuracy. We also recommend standardizing the activity before the

Table 3. Factors to multiply with standing bioimpedance to equate with lying

\begin{tabular}{ccc}
\hline $\begin{array}{c}\text { Age range } \\
\text { (years) }\end{array}$ & $\boldsymbol{N}$ & $\begin{array}{c}\text { Factor } \\
\text { (standard deviation) }\end{array}$ \\
\hline 5 to 14 & 27 & $1.031(0.012)$ \\
15 to 30 & 61 & $1.024(0.012)$ \\
31 to 59 & 73 & $1.018(0.011)$ \\
$60+$ & 44 & $1.016(0.012)$
\end{tabular}

measurement to ensure consistency. When subjects went from lying to standing, there was a small $(1 \Omega)$ difference between that measurement and the first standing measurement. Prolonged stasis is known to cause fluid shifts. This would be corrected by gentle physical activity immediately before any bioimpedance measurement, because movement-assisted venous and lymphatic drainage will help restore fluid distribution to a more balanced state.

The limitations of this study include the fact that effect of position on bioimpedance measurement was measured only at $50 \mathrm{kHz}$, that the majority of subjects were European, that the study was underpowered to detect whether ethnic differences in limb-to-torso ratios would affect the relationship, and that the correction factors recommended apply only if the position is maintained for a short time.

In the assessment of body fatness for the understanding of body composition, for the assessment of the effectiveness of lifestyle interventions, and for clinical use, it is important to have a simple, inexpensive method of measuring body composition that is practical with respect to time, comfort, and repeatability. We conclude that $50-\mathrm{kHz}$ single frequency bioimpedance may be measured with accuracy in the standing position within 2 or 3 minutes of electrode placement, and, with appropriate adjustment, population-specific validated prediction equations may be applied. 


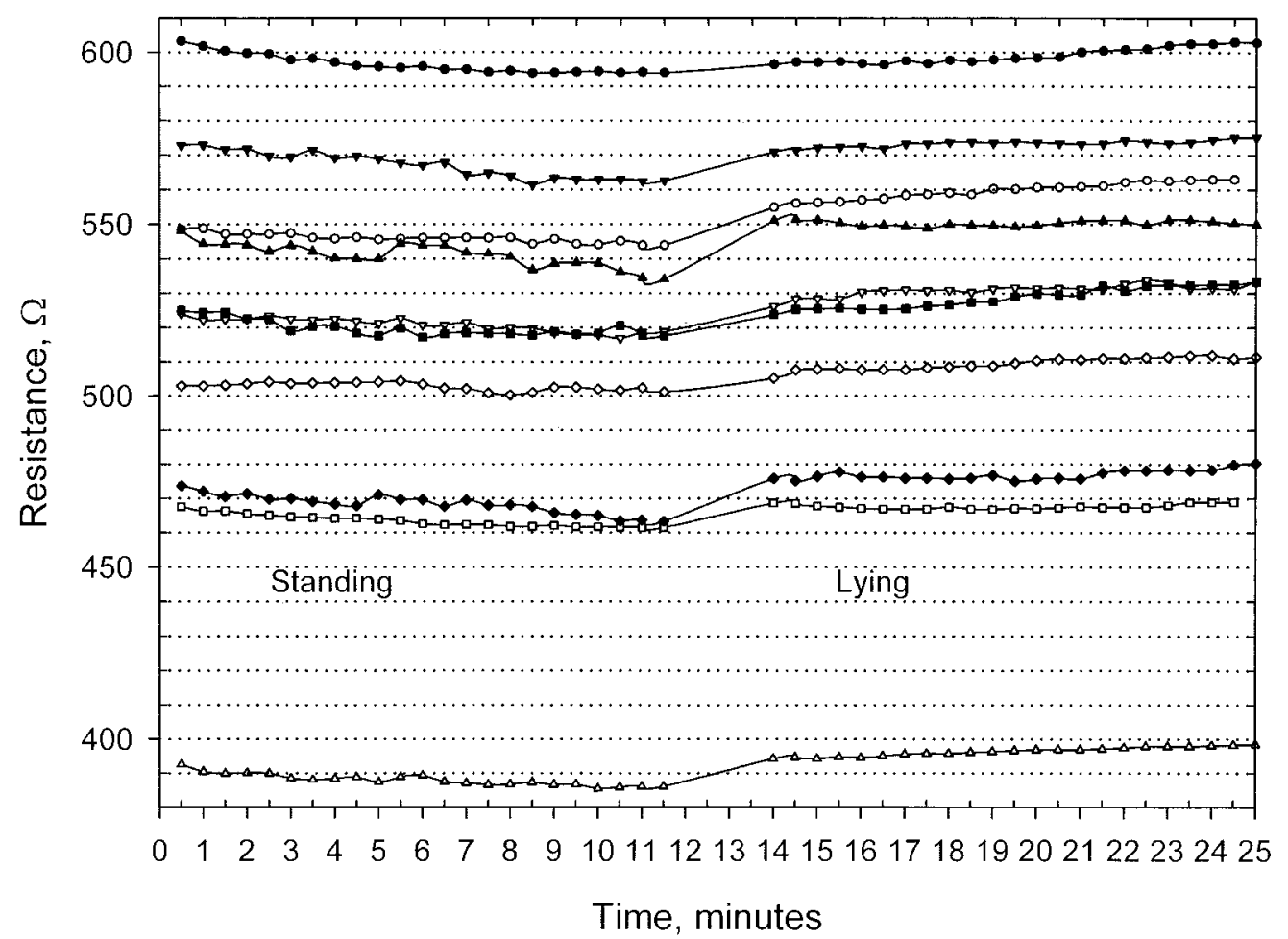

Figure 3: Relationship of resistance measurement lying and standing with time. Each symbol represents one subject.

\section{Acknowledgment}

The support of Impedimed, Queensland, Australia, for the provision of electrodes and some technical assistance is acknowledged.

\section{References}

1. NIH Consensus Statement. Bioelectrical impedance analysis in body composition measurement. National Institutes of Health Technology Assessment Conference Statement. December 12-14, 1994. Nutrition. 1996;12:749-62.

2. Kyle UG, Bosaeus I, De Lorenzo AD, et al. Bioelectrical impedance analysis - part I: review of principles and methods. Clin Nutr. 2004;23:1226-43.

3. Kyle UG, Bosaeus I, De Lorenzo AD, et al. Bioelectrical impedance analysis_-part II: utilization in clinical practice. Clin Nutr. 2004;23:1430-53.

4. Gudivaka R, Schoeller D, Kushner RF. Effect of skin temperature on multifrequency bioelectrical impedance analysis. J Appl Physiol. 1996;81:838-45.

5. Leman CR, Adeyemo AA, Schoeller DA, Cooper RS, Luke A. Body composition of children in south-western Nigeria: validation of bio-electrical impedance analysis. Ann Trop Paediatr. 2003;23:61-7.

6. Freitas IF Jr, Rush EC, Kolt G, Luke A. An alternative way of measuring hand-to-foot single frequency bioimpedance. Int J Body Comp Res. 2005;3:34.
7. Bland JM, Altman DG. Statistical methods for assessing agreement between two methods of clinical measurements. Lancet. 1986;1:307-10.

8. Kushner RF, Gudivaka R, Schoeller DA. Clinical characteristics influencing bioelectrical impedance analysis measurements. Am J Clin Nutr. 1996;64:423S-7S.

9. Fenech M, Jaffrin MY. Extracellular and intracellular volume variations during postural change measured by segmental and wrist-ankle bioimpedance spectroscopy. IEEE Trans Biomed Eng. 2004;51:166-75.

10. Zhu F, Schneditz D, Wang E, Levin NW. Dynamics of segmental extracellular volumes during changes in body position by bioimpedance analysis. J Appl Physiol. 1998;85: 497-504.

11. De Lorenzo A, Andreoli A, Deurenberg P. Impedance ratio as a measure of water shifts. Ann Nutr Metab. 1997;41:22-8.

12. Andreoli A, Melchiorri G, De Lorenzo A, Caruso I, Sinibaldi Salimei P, Guerrisi M. Bioelectrical impedance measures in different position and vs dual-energy X-ray absorptiometry (DXA). J Sports Med Phys Fitness. 2002;42: 186-9.

13. Lundvall J, Bjerkhoel $\mathbf{P}$, Quittenbaum S, Lindgren $\mathbf{P}$. Rapid plasma volume decline upon quiet standing reflects large filtration capacity in dependent limbs. Acta Physiol Scand. 1996;158:161-7.

14. Slinde F, Bark A, Jansson J, Rossander-Hulthen L. Bioelectrical impedance variation in healthy subjects during $12 \mathrm{~h}$ in the supine position. Clin Nutr. 2003;22:153-7. 